\title{
Totally laparoscopic total gastrectomy using the "enjoyable space" approach coupled with self-pulling and latter transection reconstruction versus laparoscopic-assisted total gastrectomy for upper gastric cancer: short-term outcomes
}

\author{
Xian-tu Qiu ${ }^{1}$, Chang-yue Zheng ${ }^{1}$, Ya-lun Liang ${ }^{2}$, Long-zhi Zheng ${ }^{1}$, Bin Zu ${ }^{1}$, Han-he Chen ${ }^{1}$, Zhi-yong Dong ${ }^{2}$, Li-mei Zhu' \\ Wei $\operatorname{Lin}^{1,3}$ \\ ${ }^{1}$ Department of Gastrointestinal Surgery and Gastrointestinal Surgery Research Institute, The Affiliated Hospital of Putian University, \\ Putian, Fujian, China \\ ${ }^{2}$ Department of Gastrointestinal Surgery, The First Affiliated Hospital of Jinan University, Guangzhou, China \\ ${ }^{3}$ The School of Clinical Medicine, Fujian Medical University, University Town, Fuzhou, Fujian, China
}

Videosurgery Miniinv 2022; 17 (2): 352-364 DOI: https://doi.org/10.5114/wiitm.2022.113568

\begin{abstract}
Introduction: With the development of minimally invasive surgery in recent years, totally laparoscopic total gastrectomy (TLTG) has attracted more attention.

Aim: To introduce the more comprehensive "enjoyable space" approach coupled with the self-pulling and latter transaction (SPLT) reconstruction technique to perform TLTG and investigate its safety and feasibility.

Material and methods: Ninety-seven patients with primary upper gastric cancer underwent laparoscopic radical total gastrectomy between January 2020 and December 2020. Among these patients, 46 underwent laparoscopic-assisted total gastrectomy (LATG), and 51 underwent TLTG. We compared the clinicopathological characteristics, surgical outcomes and postoperative complications between the two groups.

Results: There were no significant differences in the clinicopathological characteristics between the two groups ( $p>0.05)$. However, the TLTG group had a slightly lower mean operative time and mean blood loss than the LATG group ( $p<0.05$ each). Although there were similarities in the mean times to first flatus, liquid diet, and soft diet, the duration of hospital stay was significantly reduced in the TLTG group $(p<0.05)$. No significant differences in overall complications and E-J-related complications were found between the two groups (15.2\% vs. 25.4\%, $p>0.05)$.

Conclusions: TLTG is a safe and feasible procedure for treating upper gastric cancer. The enjoyable space approach in conjunction with SPLT reconstruction is an appropriate comprehensive technique with several advantages over LATG.
\end{abstract}

Key words: gastric cancer, laparoscopic surgery, complete mesogastrium excision, "enjoyable space" approach, self-pulling and latter transaction.

\section{Introduction}

With vast improvements in laparoscopic instruments and techniques, laparoscopy-assisted total gastrectomy (LATG) has gained widespread pop- ularity for the treatment of upper gastric cancer (UGC) because of its well-known advantages. LATG is a minimally invasive procedure with favorable cosmetic outcomes and a faster recovery time than its

Address for correspondence

Li-mei Zhu MD, Wei Lin MD, PhD, Department of Gastrointestinal Surgery and Gastrointestinal Surgery Research Institute, the Affiliated Hospital of Putian University, 999 Dongzhen Road, Putian 351100, Fujian, China, phone: +86-0594-2730373, fax: +86-0594-2293910,

e-mail: 137143598@qq.com; linwbj@outlook.com 
open counterpart [1-4]. However, totally laparoscopic total gastrectomy (TLTG) is not yet widely adopted in clinical practice. As a technical extension of laparoscopic gastrectomy, the main concerns surrounding TLTG involve two aspects: appropriate intracorporeal digestive tract reconstruction and inherent oncological safety.

Intracorporeal digestive tract reconstruction is a complicated TLTG procedure. Moreover, since intracorporeal esophagojejunostomy (IEJ) is performed in a high anastomotic site and narrow operating space, it is still regarded as a more challenging aspect $[5,6]$ Although the overlap and functional end-to-end anastomosis (FETE) methods have been indicated to be feasible and safe, to date, there is no consensus on the optimal intracorporeal digestive tract reconstruction method because of the low efficiency and technical complexity of the procedure [7-9]. However, Hong et al. [10] reported a novel reconstructive method of self-pulling and latter transection (SPLT), which seems to be a convenient and promising procedure. Nevertheless, the superiority of techniques such as TLTG-SPLT reconstruction compared to LATG has not been effectively evaluated.

Radical resection of the primary lesion and relative lymph nodes (LNs) in LATG is a common major oncological safety concern. Although D2 lymphadenectomy has been recommended to treat advanced gastric cancer, locoregional recurrence often occurs in patients who receive radical $\mathrm{RO}$ resection [11, 12]. Furthermore, some scholars believe that this phenomenon might be strongly associated with minimal residual disease or potential cancer cell dissemination during the operation [13, 14]. Although the prognosis of colorectal cancer has significantly improved due to the widespread application of complete mesenteric excision, the concept and importance of complete mesogastrium excision (CME) have not yet been widely recognized and effectively evaluated in curative gastrectomy [15-18]. Our previous study presented the "enjoyable space" approach to achieve D2 + CME and confirmed its feasibility and safety in the LATG procedure [19]. However, this technique has not yet been further appraised in TLTG. Moreover, regarding the use of SPLT reconstruction in TLTG and the above concerns, the oncological safety of IEJ should be evaluated urgently because of the lack of intraoperative frozen sections for determining the upper margin of specimens, especially when encountering tumors with high-level esophageal invasion.

\section{Aim}

Thus, this study aimed to assess the short-term outcomes of TLTG using the comprehensive enjoyable space approach with SPLT reconstruction and to investigate its safety and feasibility relative to LATG.

\section{Material and methods}

\section{Patients and inclusion/exclusion criteria}

We retrospectively collected the data of 97 patients who underwent total gastrectomy by LATG (51 patients) and TLTG (46 patients) for UGC at our hospital over the period of January to December 2020. All patients were subjected to preoperative assessments, including endoscopic examination and endoscopic biopsy specimen analysis, computed tomography (CT) scanning, and abdominal ultrasonography (US). The tumor depth (T), nodal status (N) and tumor stage (TNM) were determined according to the guidelines of the eighth edition of the American Joint Committee on Cancer (AJCC) staging manual [20]. The inclusion criteria were as follows: preoperative examinations confirming UGC; no preoperative evidence of distant metastasis; invasion of the lower esophagus no more than $2 \mathrm{~cm}$ above the cardia; surgery performed by the same surgeon; and curative resection (RO) according to the postoperative pathological diagnosis. The exclusion criteria were as follows: prior abdominal surgery or preoperative chemoradiation therapy; preoperatively or intraoperatively proven distant metastasis; stage T4b disease; esophageal invasion more than $2 \mathrm{~cm}$ above the cardia; combined resection; and missing pathological data. Patients with preoperative or intraoperative severe LN metastases or peripheral organ involvement who underwent open surgery were also excluded from this study. In addition, clinicopathological characteristics, surgical outcomes and postoperative complications were postoperatively collected and analyzed according to the surgical video and complete medical records. Written informed consent was obtained after a detailed explanation of the procedures' benefits and risks was provided to both the patients and their families. This study (approval number: 202136) was approved by the Ethics Committee of our hospital. 


\section{Surgical technique}

\section{Position and trocar placements}

The patients were placed in the reverse Trendelenburg position with their heads elevated at approximately 15 to $20^{\circ}$. The surgeon stood on the patient's left side, with the assistant on the opposite side, while the camera operator stood between the patient's legs. For laparoscope insertion, a 10-mm trocar was inserted into a curved incision at the upper right side of the umbilicus for laparoscopy. Then, two 12-mm trocars were inserted into the left preaxillary line $2 \mathrm{~cm}$ below the costal margin and the left midclavicular line $2 \mathrm{~cm}$ above the umbilicus as the major hand ports for the surgeon and the assistant, and two 5-mm trocars were inserted into their contralateral sites for assistance and exposure.

The enjoyable space approach and SPLT reconstruction have been reported previously in the literature $[10,19]$. The procedure can be briefly described as follows.
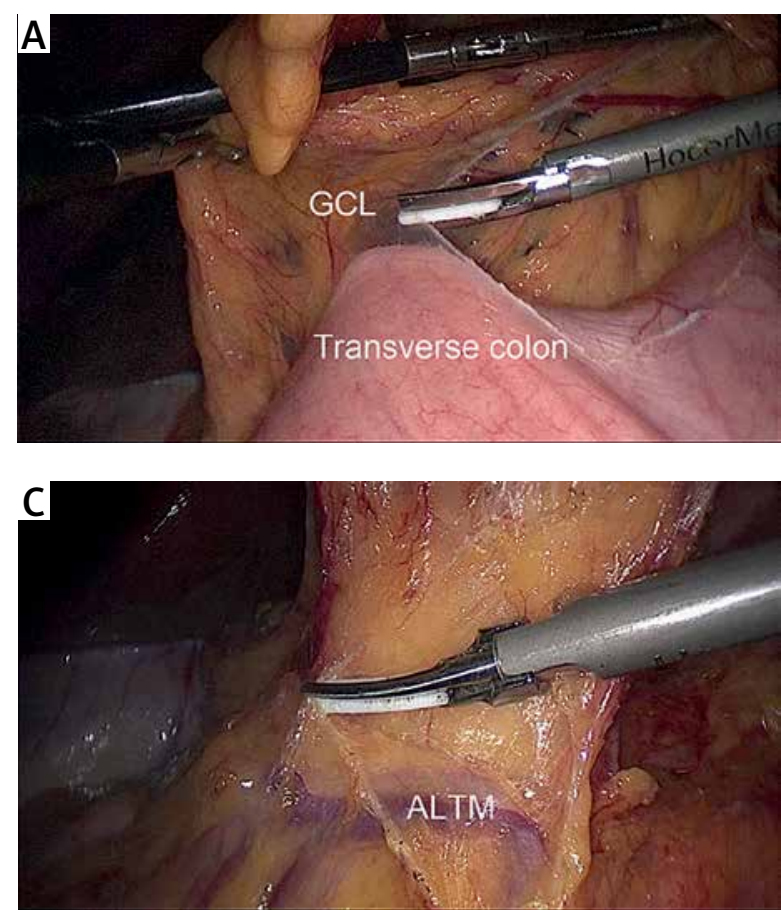

The enjoyable space approach for D2 + CME total gastrectomy

Step 1. Separating the inferior region: The gastrocolic ligament was divided routinely along the border of the transverse colon toward the left, exposing the tail of the pancreas and the retro-pancreatic space toward the right to locate the gastrocolic fusional fascia (Photos $1 \mathrm{~A}, \mathrm{~B}$ ). Then, the surgical plane was extended along the anterior lobe of the transverse mesocolon (ALTM) to expose and divide the right gastroepiploic vein (RGEV) and the right gastroepiploic artery (RGEA) (LN No. 6) at their origin (Photos 1 C-E). Subsequently, the anterior pancreatic fascia (APF) was gently peeled toward the superior border of the pancreas, exposing the gastroduodenal artery (GDA), the common hepatic artery (CHA) and the origin of the right gastric artery (RGA) (Photos $1 \mathrm{~F}, \mathrm{G}$ ).

Step 2. Separating the superior recess: The main vascular networks within the superior recess were used as indicators of exposure and vascularization to maintain fascial integrity. Prior to the vascular-
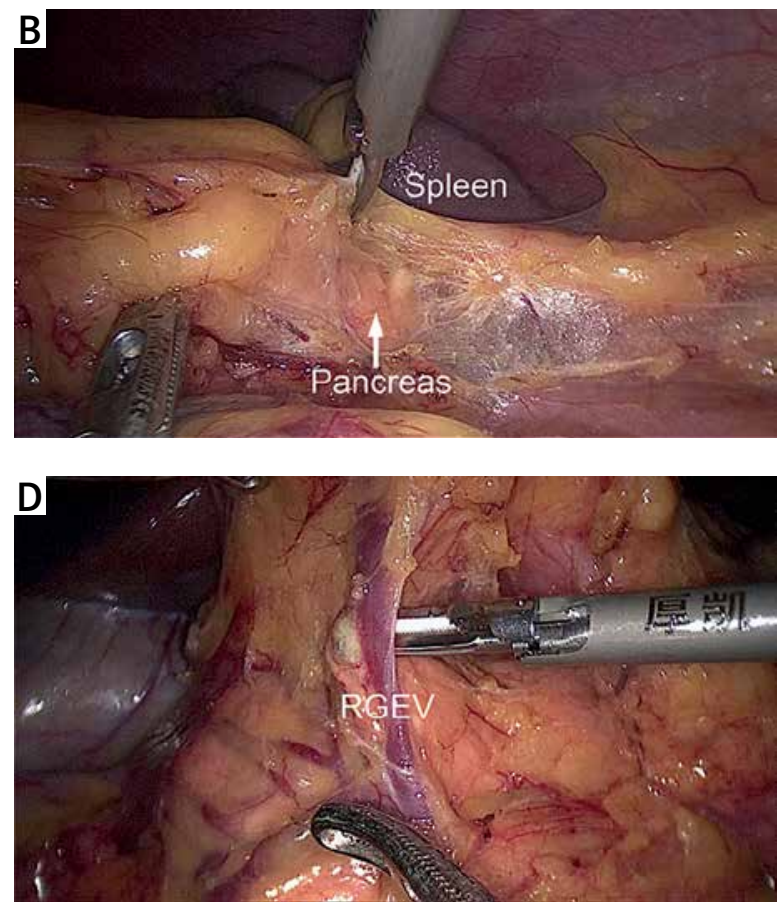

Photo 1. Intraoperative image showing the inferior region and the superior recess separation procedure. A - Dividing the gastrocolic ligament. B - Exposing the tail of the pancreas and the retropancreatic space. $\mathbf{C}$ - Locating and extending the incision along the anterior lobe of the transverse mesocolon. D - Exposing the right gastroepiploic vein

$G C L$ - gastrocolic ligament, ALTM - anterior lobe of the transverse mesocolon, RGEV - right gastroepiploic vein, RGEA - right gastroepiploic artery, $G D A$ - gastroduodenal artery, APF - anterior pancreatic fascia, CHA - common hepatic artery, RGA - right gastric artery, PHA - proper hepatic artery, $P V$ - portal vein, $L G A$ - left gastric artery. 

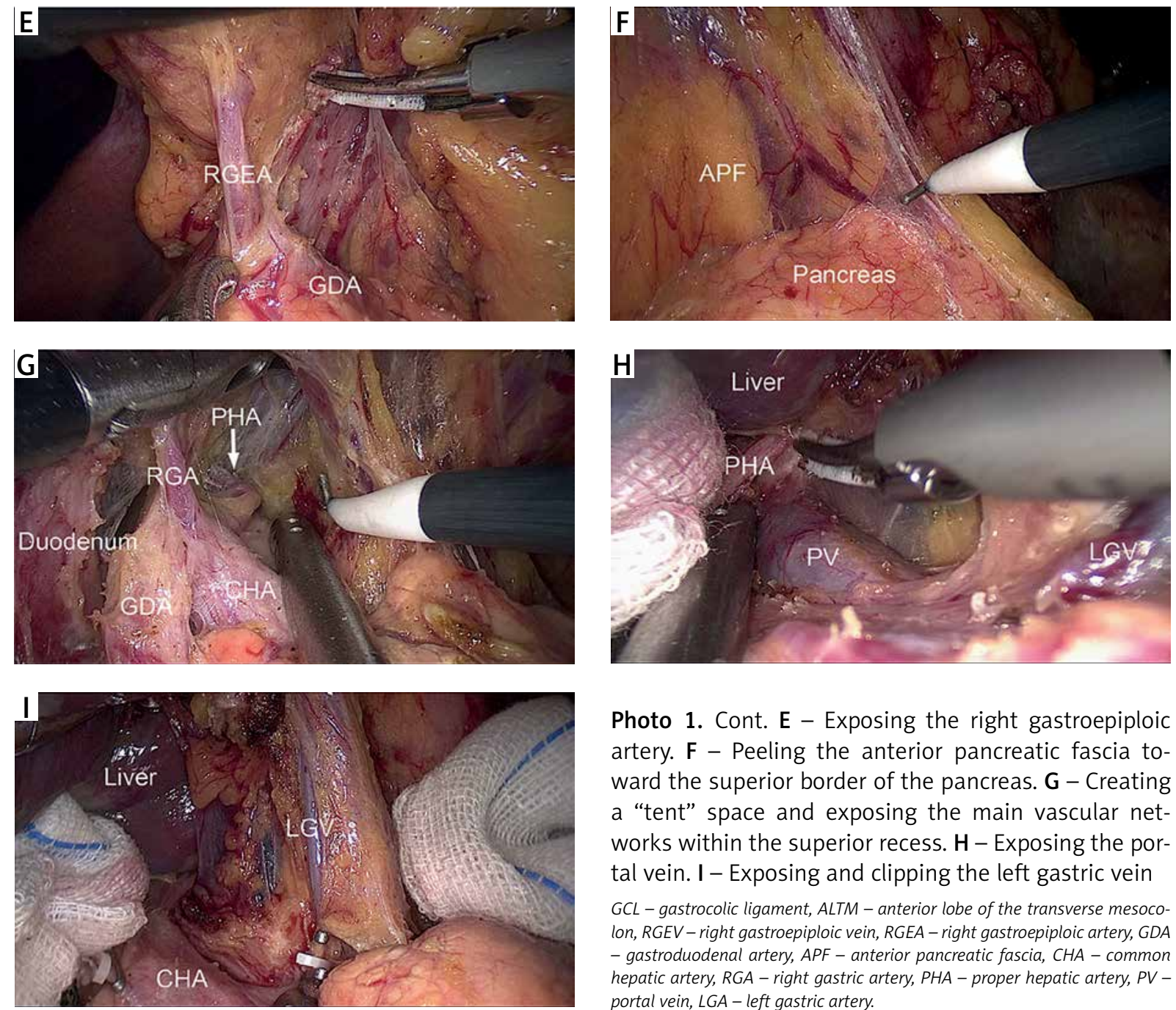

Photo 1. Cont. E - Exposing the right gastroepiploic artery. $\mathbf{F}$ - Peeling the anterior pancreatic fascia toward the superior border of the pancreas. G - Creating a "tent" space and exposing the main vascular networks within the superior recess. $\mathrm{H}$ - Exposing the portal vein. I - Exposing and clipping the left gastric vein

$G C L$ - gastrocolic ligament, ALTM - anterior lobe of the transverse mesocolon, $R G E V$ - right gastroepiploic vein, RGEA - right gastroepiploic artery, GDA - gastroduodenal artery, APF - anterior pancreatic fascia, CHA - common hepatic artery, RGA - right gastric artery, PHA - proper hepatic artery, PV portal vein, $L G A-$ left gastric artery.

ization and division of the RGA (LN No. 5), a "tent" space was created by peeling the perigastric fascia from the surface of the GDA and CHA (LN No. 8a) and holding up the posterior gastric wall. Next, the anterior region of the hepatoduodenal ligament (HDL) (LN No. 12a) was easily peeled along the proper hepatic artery (PHA) and portal vein (Photos $1 \mathrm{G}, \mathrm{H}$ ). Then, the left gastric vein was exposed and clipped at its point of origin (Photo $1 \mathrm{l}$ ).

Step 3. Separating the splenic recess: The loose connective tissue within the origin of the left gastric artery (LGA) and splenic artery (SPA) was cut to gain access to the splenic recess and to locate the left Gerota's fascia (Photo 2 A). Next, the dissection was meticulously continued along the left Gerota's fascial surface to expand the "enjoyable space": to the lower region to expose the SPA; to the left to re-

veal the posterior edge of the middle-upper spleen; to the right to denude the right crus of the diaphragm (RCD); and to the upper region to expose the esophageal hiatus $(E H)$ and the gastrophrenic ligament (GPL) (Photos $2 \mathrm{~B}-\mathrm{F})$. Thus, the perigastric space was established to complete the whole dissection of the perigastric mesogastrium (Photo $2 \mathrm{G}$ ). Then, the celiac trunk (LN No. 9) and the LGA (LN No. 7) were completely skeletonized, and the LGA was divided at its root (Photo $2 \mathrm{H}$ ). The surrounding mesenteries and the gastrosplenic ligament (GSL) were successively pulled up to expose the splenic vessels and their branches. The left gastroepiploic artery, posterior gastric artery (PGA), and all short gastric vessels were divided, and the corresponding LNs were removed (LN No. 4sa and 4sb) (Photos $2 \mathrm{I}-\mathrm{K}$ ). The fatty lymphatic tissues were also completely removed along 
the splenic vessels (LN No. 11) and around the splenic hilum (LN No. 10), easily exposing the cut-away GPL (Photo $2 \mathrm{~L}$ ). At the lesser curvature, the hepatogastric ligament (HGL) was dissected along the lower border of the liver, and the LNs around the lesser curvature (No. 3) were removed. Finally, the phrenoesophageal membrane and both vagus nerves were divided to dissect LNs No. 1 and No. 2 and expose the lower
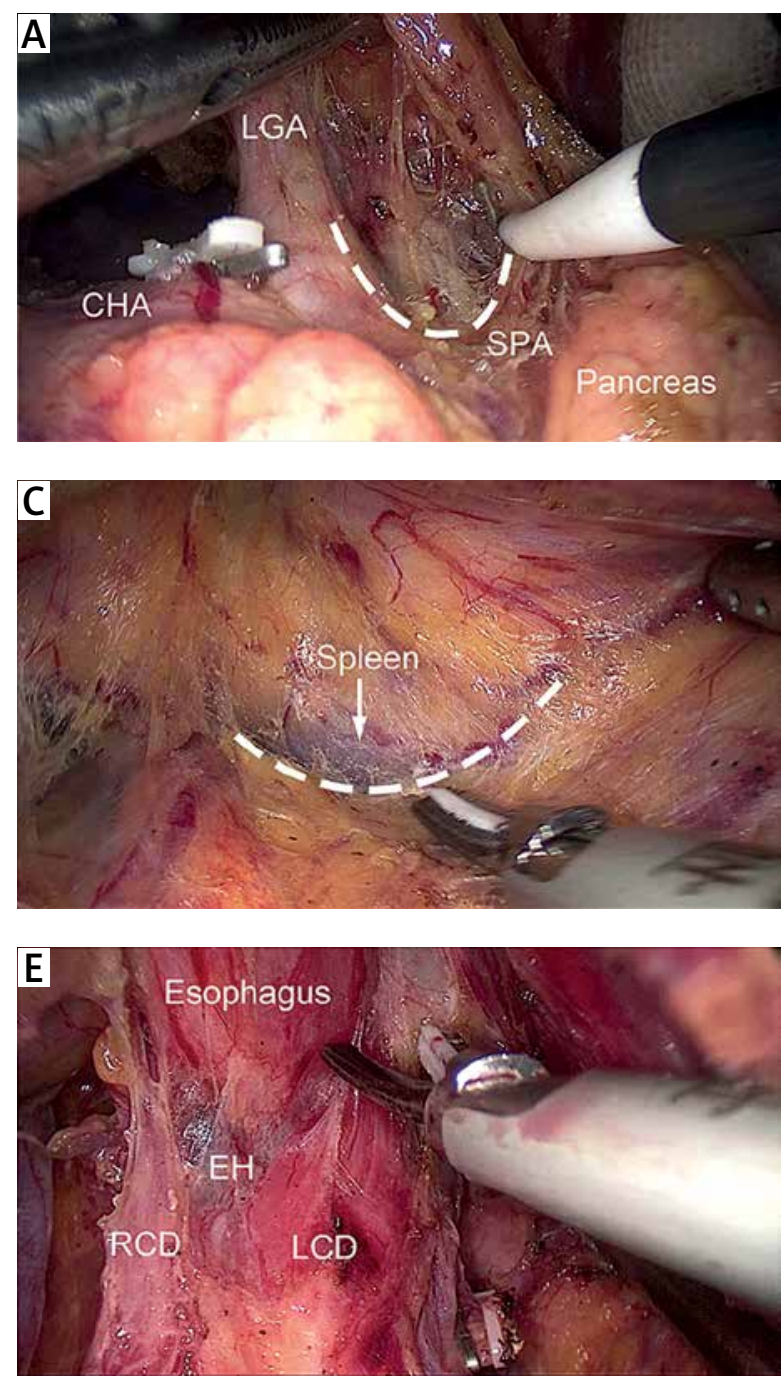

esophagus. This step completed the enjoyable space approach procedure, and the duodenum was transected with the first linear stapler (LS).

\section{The reconstruction procedure of SPLT}

Prior to digestive tract reconstruction, the cardia (or the lower esophagus above the upper margin of the tumor) was tightly ligated with a sterilized rope.
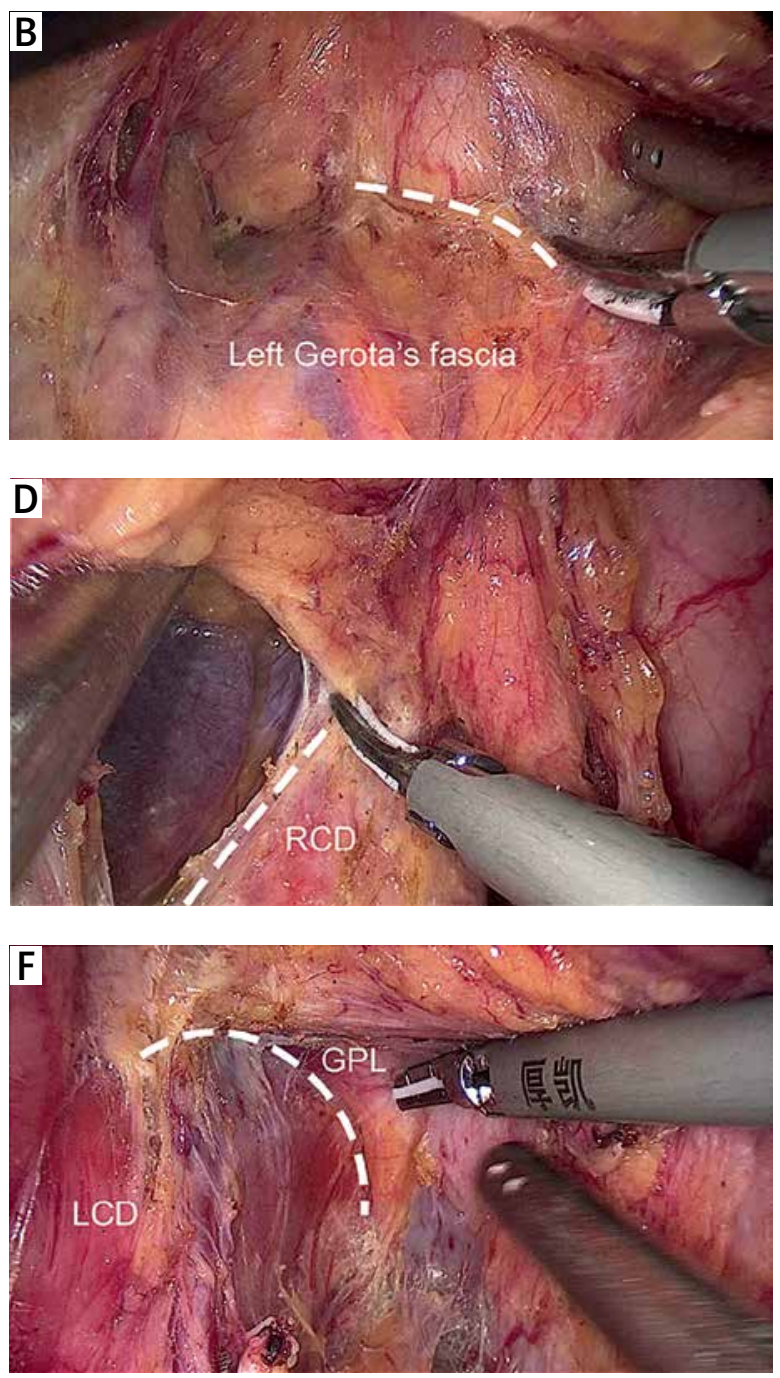

Photo 2. Intraoperative image showing the splenic recess separation procedure and the spleen-preserving splenic hilar lymphadenectomy separation procedure. A - Exposing the entrance; the dashed line highlights the entrance pathway. B - Locating the left Gerota's fascia and separating the lower border's pathway; the dashed line shows the pathway of the lower border. C - Exposing the posterior edge of the middle-upper spleen along the left Gerota's fascia; the dashed line shows the left border pathway. D - Denuding the right crus of the diaphragm; the dashed line shows the right border pathway. E-Exposing the esophageal hiatus. $\mathbf{F}$ - Exposing the upper border; the dashed line shows part of the upper border pathway

LGA - left gastric artery, CHA - common hepatic artery, SPA - splenic artery, EH - esophageal hiatus, RCD - right crus of the diaphragm, LCD - left crus of the diaphragm, GPL - gastrophrenic ligament, PGA - posterior gastric artery, LGEVs - left gastroepiploic vessels, ISLVs - inferior splenic lobar vessels, SSLVs - superior splenic lobar vessels, SGA - short gastric artery. 

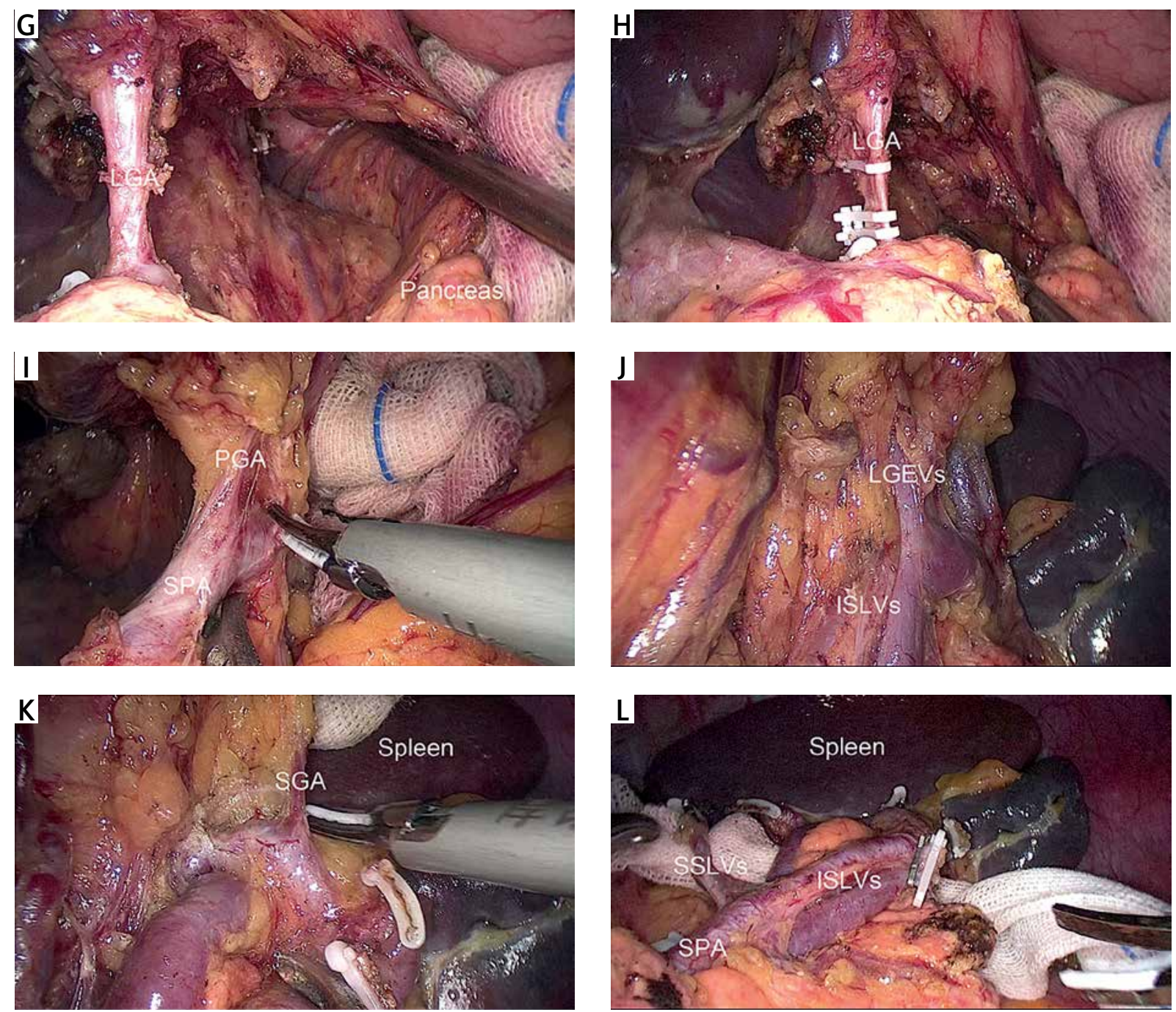

Photo 2. Cont. G - Showing the overall view of the "enjoyable space". H - Skeletonizing and clipping the left gastric artery. I - Skeletonizing the splenic artery and separating the posterior gastric artery. J - Exposing the left gastroepiploic vessels. $\mathbf{K}$ - Exposing the short gastric artery. $\mathbf{L}$ - Overall view of the splenic hilum after lymphadenectomy

LGA - left gastric artery, CHA - common hepatic artery, SPA - splenic artery, EH - esophageal hiatus, RCD - right crus of the diaphragm, LCD - left crus of the diaphragm, GPL - gastrophrenic ligament, PGA - posterior gastric artery, LGEVs - left gastroepiploic vessels, ISLVs - inferior splenic lobar vessels, SSLVs - superior splenic lobar vessels, SGA - short gastric artery.

Next, by pulling the ligature rope to a different level, the lower esophagus could be easily denuded and pulled down from the posterior mediastinum, creating room for anastomosis. Then, a hole was made $2-3 \mathrm{~cm}$ above the ligature rope on the posterior wall of the esophagus. After making an intestinal mesenteric hole on the opposite side, to create an entrance for a second LS, another hole was punched at the jejunum's anti-mesenteric border, $20-25 \mathrm{~cm}$ distal to the ligament of Treitz (Photo $3 \mathrm{~A}-\mathrm{C}$ ). Thus, a side-to-side esophagojejunostomy (E-J) was established through two holes, forming a common opening (Photo $3 \mathrm{D}$ ). After carefully checking the E-J anastomosis, the esophagus and the afferent loop jejunum were simultaneously transected to close the entry hole with a third LS (Photos $3 \mathrm{E}, \mathrm{F})$. Subsequently, a side-to-side jejunojejunostomy (J-J) similar to FETE was performed between the afferent loop stump and the efferent loop 40-50 cm below the E-J with another two LSs (Photos $3 \mathrm{G}-1$ ). Then, the reconstruction procedure of SPLT was completed with the whole excision of the specimen, which was then placed into a specimen bag. 

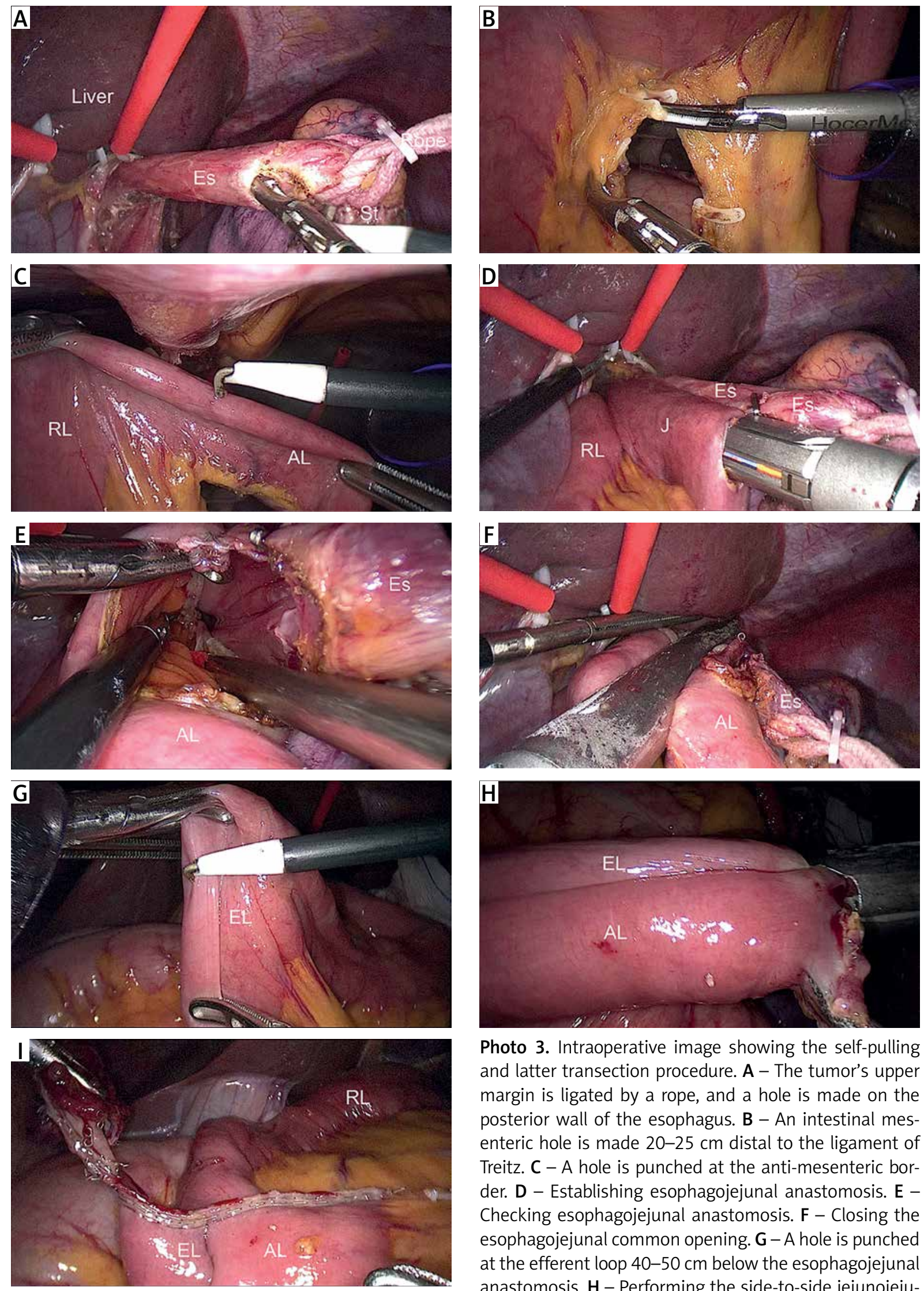

Photo 3. Intraoperative image showing the self-pulling and latter transection procedure. A - The tumor's upper margin is ligated by a rope, and a hole is made on the posterior wall of the esophagus. B - An intestinal mesenteric hole is made $20-25 \mathrm{~cm}$ distal to the ligament of Treitz. C - A hole is punched at the anti-mesenteric border. D - Establishing esophagojejunal anastomosis. E Checking esophagojejunal anastomosis. F - Closing the esophagojejunal common opening. $\mathbf{G}-\mathrm{A}$ hole is punched at the efferent loop 40-50 $\mathrm{cm}$ below the esophagojejunal anastomosis. $\mathbf{H}$ - Performing the side-to-side jejunojejunostomy. I - Closing the jejunojejunal common opening

Es - esophagus, St - stomach, J-jejunum, RL - roux limb, AL - afferent loop, $E L-$ efferent loop. 
In the LATG group, gastric transection and Rouxen-Y reconstruction using a circular stapler (CS) were performed through an epigastric mini-laparotomy. In contrast, the incision of the TLTG group, which was required to remove the specimen, was only $3 \mathrm{~cm}$ (according to the size of the tumor) and extended from the umbilical trocar site.

\section{Statistical analysis}

All statistical analyses were performed using IBM SPSS Statistics version 20 (SPSS Inc., Chicago, IL, USA; IBM Corp., Armonk, NY, USA), and the data are reported as the means \pm standard deviations (SDs). The $\chi^{2}$ test or Fisher's exact test was used

Table I. Comparisons of clinicopathological characteristics between the groups

\begin{tabular}{|c|c|c|c|}
\hline Variable & TLTG group $(n=46)$ & LATG group $(n=51)$ & $P$-value \\
\hline Sex, $n$ : & & & 0.827 \\
\hline Male & 31 & 36 & \\
\hline Female & 15 & 15 & \\
\hline Age [years] & $63.3 \pm 9.1$ & $63.9 \pm 8.2$ & 0.712 \\
\hline $\mathrm{BMI}\left[\mathrm{kg} / \mathrm{m}^{2}\right]$ & $23.74 \pm 3.98$ & $23.07 \pm 4.18$ & 0.424 \\
\hline Tumor location, $n$ : & & & 0.939 \\
\hline Cardia & 31 & 34 & \\
\hline Body & 15 & 17 & \\
\hline Tumor size $[\mathrm{cm}]$ & $3.6 \pm 1.8$ & $4.3 \pm 2.4$ & 0.136 \\
\hline PRML of UGC & $2.67 \pm 1.04$ & $2.59 \pm 0.89$ & 0.671 \\
\hline PRML of cardia tumor & $2.20 \pm 0.18$ & $2.19 \pm 0.17$ & 0.898 \\
\hline Tumor depth (pT): & & & 0.989 \\
\hline pT1 & 5 & 6 & \\
\hline pT2 & 8 & 8 & \\
\hline pT3 & 22 & 26 & \\
\hline pT4a & 11 & 22 & \\
\hline Lymph node metastasis (pN): & & & 0.523 \\
\hline pNO & 19 & 17 & \\
\hline pN1 & 8 & 14 & \\
\hline $\mathrm{pN} 2$ & 13 & 11 & \\
\hline pN3 & 6 & 9 & \\
\hline TNM stage, $n$ : & & & 0.998 \\
\hline IA & 5 & 6 & \\
\hline IB & 6 & 5 & \\
\hline$\| A$ & 6 & 7 & \\
\hline$\| B$ & 9 & 10 & \\
\hline$\| I I A$ & 11 & 14 & \\
\hline IIIB & 7 & 6 & \\
\hline IIIC & 2 & 3 & \\
\hline
\end{tabular}

$B M I$ - body mass index, PRML - proximal resection margin length, UGC - upper gastric cancer. 
to compare categorical variables, while unpaired Student's $t$-test was used to compare continuous variables depending on the distribution of the data. $P$-values less than 0.05 were considered statistically significant.

\section{Results}

\section{Clinicopathological characteristics of the included patients}

The clinicopathological characteristics of the 97 patients are presented in Table I. The cohort consisted of 67 (69.0\%) males and 30 (31.0\%) females with a mean age of $63.7 \pm 8.6$ years (range: $35-84$ years). The mean body mass index (BMI) was $23.39 \pm 4.08$ $\mathrm{kg} / \mathrm{m}^{2}$ (range: $16.90-34.72 \mathrm{~kg} / \mathrm{m}^{2}$ ), and the mean tumor size was $4.0 \pm 2.2 \mathrm{~cm}$ (range: $1.0-15.0 \mathrm{~cm}$ ). The mean lengths of the proximal resection margin for UGC and cardia tumors were $2.62 \pm 0.96 \mathrm{~cm}$ (range: $1.9-7.0 \mathrm{~cm}$ ) and $2.19 \pm 0.18 \mathrm{~cm}$ (range: $1.9-2.7 \mathrm{~cm}$ ), respectively. No significant differences were observed in sex, age, BMI, tumor location, tumor size, tumor depth (pT), LN metastasis (pN), TNM stage, or proximal resection margin length ( $p>0.05$ each).

\section{Surgical outcomes of the TLTG group vs. the LATG group}

All patients successfully underwent laparoscopic radical total gastrectomy (with TLTG or LATG), none of the patients required conversion to laparotomy, and no operation-related deaths occurred during the perioperative period. The mean operative time for all 97 patients was $228.0 \pm 29.5$ min (range: $160-310$ $\mathrm{min})$, while the mean blood loss was $73.8 \pm 29.3$ $\mathrm{ml}$ (range: $30-180 \mathrm{ml}$ ), and the median number of retrieved LNs was $34.3 \pm 7.4$ (range: 26-64) per patient. The mean operative time and the mean blood loss were slightly reduced in the TLTG group $(p<$ 0.05 each). Although the mean times to first flatus, liquid diet, and soft diet were similar, the duration of hospital stay was significantly reduced in the TLTG group $(p<0.05)$ (Table II).

\section{Intraoperative and postoperative complications of the TLTG group vs. the LATG group}

The intraoperative and postoperative complications of both groups are detailed in Table III. The

Table II. Comparisons of surgical outcomes between the groups

\begin{tabular}{|lccc|}
\hline Variable & TLTG group $(n=46)$ & LATG group $(n=51)$ & $P$-value \\
\hline Operation time [min] & $221.6 \pm 26.2$ & $233.8 \pm 31.2$ & 0.041 \\
\hline Blood loss [ml] & $67.6 \pm 28.7$ & $79.4 \pm 29.0$ & 0.047 \\
\hline Mean no. of retrieved LNs, $n$ & $33.9 \pm 7.8$ & $34.5 \pm 7.1$ & 0.688 \\
\hline Time to first flatus [days] & $3.1 \pm 0.9$ & $3.3 \pm 1.2$ & 0.314 \\
\hline Time to liquid diet [days] & $3.2 \pm 1.0$ & $3.5 \pm 1.5$ & 0.279 \\
\hline Time to soft diet [days] & $6.0 \pm 1.0$ & $6.2 \pm 1.5$ & 0.674 \\
\hline Hospital stay [days] & $8.0 \pm 1.4$ & $9.5 \pm 2.0$ & 0.001 \\
\hline
\end{tabular}

Table III. Comparisons of intraoperative and postoperative complications between the groups

\begin{tabular}{|lccc|}
\hline Variable & TLTG group $(n=46)$ & LATG group $(n=51)$ & $P$-value \\
\hline Pulmonary infection $(n)$ & 5 & 7 & \\
\hline Paralytic ileus $(n)$ & 1 & 1 & \\
\hline Abdominal infection $(n)$ & 0 & 3 & 0.684 \\
\hline E-J-related complications $(n):$ & 1 & 0 & \\
\hline Submucosal anastomosis & 1 & 2 & \\
\hline Anastomotic stenosis & 0 & 1 & 0.315 \\
\hline Anastomotic hemorrhage & 0 & 13 & \\
\hline Total complications $(n)$ & 7 & & \\
\hline
\end{tabular}


overall intraoperative and postoperative morbidity rate among all patients was 20.6\% (20/97), while the E-J-related complications and the overall complications did not differ significantly between the TLTG group and the LATG group $(2.1 \%(1 / 46)$ vs. $5.8 \%$ (3/51), $p>0.05$; $15.2 \%$ (7/46) vs. $25.4 \%$ (13/51), $p>0.05)$. In addition, one patient with submucosal anastomosis in the TLTG group was treated by incising the esophageal mucosa to keep the anastomosis unobstructed. Other patients with postoperative complications were successfully treated conservatively and discharged, and the mortality rate in both groups was $0 \%$.

\section{Follow-up}

Of all 97 patients, 95 (97.9\%) patients had followups ranging from 9 to 20 months (median: 14 months). A total of 45 patients in the TLTG group (96.3\%, median: 14 months, range: 9-20 months) and $50 \mathrm{pa}$ tients in the LATG group (97.6\%, median: 14 months, range: 9-20 months) underwent these follow-ups. Furthermore, 3 patients presented with postoperative tumor metastasis due to advanced tumors, including 2 patients in the TLTG group and 3 patients in the LATG group; however, there were no deaths during the follow-up period.

\section{Discussion}

TLTG-SPLT reconstruction is an efficient and minimally invasive technique to achieve intracorporeal digestive tract reconstruction. In the present study, we adopted SPLT reconstruction to perform intracorporeal digestive tract reconstruction in the TLTG group. We found that the mean intraoperative time and intraoperative blood loss were slightly lower in the TLTG group than in the LATG group, without increasing severe complications. Since the radical gastrectomy procedure was performed in a similar manner in both groups, we believed that the improvement in surgical outcomes was mainly attributed to the application of SPLT reconstruction. In LATG, stomach resection and anastomosis should be performed through an epigastric auxiliary incision. Sometimes, this procedure is relatively difficult and requires an extension of the incision because of the limited angle of direct view (especially in obese patients), a large anteroposterior diameter, or an acute subcostal angle [21, 22]. Moreover, the extensive traction required for full exposure may cause injury to surrounding structures and unexpected bleeding, increasing the risk of anastomotic leakage. However, in TLTG using SPLT reconstruction, the whole digestive tract reconstruction procedure was mostly accomplished within a relatively wide upper abdominal cavity and was rarely affected by individual physical differences, thus avoiding injury to the surrounding tissues. Moreover, the procedure differs significantly from other methods that first transect the esophagus and jejunum. When performing SPLT reconstruction, the lower esophagus was initially pulled down with a ligature rope above the cardia or upper tumor margin. After adequately dissociating and mobilizing the lower esophagus, two holes were made at the corresponding sites of the esophagus and jejunum. In this way, the assistant could easily drag down the lower esophagus in different directions and maintain the appropriate tension. Furthermore, the surgeon can also leisurely lift the jejunum and smoothly complete the IEJ without repeatedly estimating the anastomotic angle and positioning the jejunum, thereby reducing the difficulty of creating an E-J and improving the consistency of the operation. Moreover, the E-J entry hole remained between the specimen and the afferent loop intestine after transecting the esophagus and small intestine to close the common opening. It can be easily located and serviced as one of the entrances of the subsequent J-J, further accelerating the anastomosis efficiency. Therefore, TLTG using SPLT reconstruction categorically reduces the difficulty and risk of the operation. In addition, TLTG requires only a smaller periumbilical incision for specimen acquisition, and the digestive tract reconstruction is performed in tension-free circumstances. Thus, less trauma and traction are likely to contribute to decreasing intraoperative blood loss. Consistent with our findings, previous studies have reported associations of reduced intraoperative blood loss and incision pain with a better postoperative quality of life [23]. We observed significantly shorter postoperative hospital stays in the TLTG group than in the LATG group, confirming that the minimally invasive effect during TLTG can accelerate postoperative recovery.

Intraoperative oncological safety is a critical assessment of TLTG using SPLT for the treatment of gastric cancer. Since the perigastric mesogastrium plays an important role in "metastasis V" and contains the supporting vascular, nerve and lymphatic systems of the posterior wall of the stomach, radical resection 
of gastric cancer is insufficient if only the related $\mathrm{LNS}$ are dissected $[24,25]$. CME should be conducted to remove the primary lesion and the adjacent soft tissue to achieve oncological en bloc resection. Only in this manner can locoregional recurrence caused by potential tumor dissemination and remnants be effectively prevented to achieve a radical cure effect. In the current study, the enjoyable space approach was adopted to perform a radical total gastrectomy procedure in both groups. Our data showed that the mean number of harvested LNs, which served as an objective oncological safety indicator, was sufficient and equivalent between the two groups (33.9 \pm 7.8 vs. $34.5 \pm 7.1)$ and exceeded both the pathologically evaluated and preferred levels (16 and 30, respectively) [26]. Moreover, the whole procedure was performed within the potential intrafascial spaces, providing a safe and sequential surgical plane and improving the accuracy and efficiency of the operation. As a result, none of the patients required conversion to laparotomy due to intraoperative injury or hemorrhage. Therefore, the enjoyable space approach appears to be a stable and reliable technique of total radical gastrectomy, leading to improved oncological safety and a reduced operative risk. However, due to the lack of an intraoperative frozen section to determine the specimen's proximal resection margin, the oncological safety of IEJ procedures remains a concern in TLTG-SPLT reconstruction. According to our practice, the ligature rope above the tumor margin can prevent the tumor from spreading out when performing SPLT reconstruction. The lower esophagus can easily be mobilized and separated to 8-10 cm above the cardia. Theoretically, it can provide an adequate, safe distance for anastomosis. For an accurate evaluation, we compared the length of the tumors' proximal resection margin, located in the cardia, with esophageal invasion less than $2 \mathrm{~cm}$ between the TLTG group and the LATG group in a preliminary study. Our data showed that this parameter was not significantly different between the two groups (UGC: $2.67 \pm 1.04$ vs. $2.59 \pm 0.89$; cardia tumor: $2.20 \pm 0.18$ vs. $2.19 \pm 0.17$ ), indicating the oncological safety of SPLT reconstruction in the treatment of UGC. However, preoperative or intraoperative gastroscopic positioning is recommended to obtain a negative margin for patients with obvious esophageal invasion (especially $>2 \mathrm{~cm}$ ).

IEJ-related complications are an important topic in terms of using digestive reconstruction methods in
TLTG. Previous studies have revealed that the rate of postoperative IEJ-related complications ranged from $0 \%$ to $11.7 \%$ [27-29]. The majority of the previous comparisons of IEJ-related complications between LS and CS approaches involved retrospective studies with small sample sizes; thus, the results are inconsistent and even contradictory [5, 30-32]. Among these studies, some revealed no difference in the E-J-related complication rate between the different methods. However, Murakami et al. conducted a multicenter retrospective comparative study on LS and CS following TLTG, and reported that the incidence of anastomotic stenosis in the LS group was significantly lower than that in the CS group (1.5\% vs. $7.1 \%$, $p=0.011)$, and no postoperative anastomotic bleeding was observed in the LS group ( $0 \%$ vs. $2.0 \%, p=$ 0.123 ) [28]. In agreement with previous studies, our outcomes did not show any differences in the rate of overall complications and E-J-related complications between the groups. However, we observed 3 cases of postoperative E-J-related complications, including 2 cases of anastomotic stenosis (3.92\%) and 1 case of anastomotic hemorrhage (1.96\%), all of which occurred in the LATG group with a CS. This seemingly contradictory result may be due to the insufficient number of enrolled cases. In addition, there are several possible explanations related to the favorable outcomes obtained via IEJ with an LS. First, it is recognized that IEJ with an LS can create a larger anastomosis than IEJ with a CS, thereby avoiding E-J-associated anastomotic stenosis. According to our experience, a $45 \mathrm{~mm}$ LS was usually adequate to fulfill the majority of practical needs. However, to avoid anastomosis restriction and promote safety, a $60 \mathrm{~mm}$ LS that can produce a larger anastomosis without being restricted by the diameter of the esophagus may be more suitable. In addition, the diameter and smoothness of the anastomosis would be further ensured by minimizing the removal of esophageal and jejunal tissues when safely closing the entry hole. Second, IEJ was completed in a tension-free condition, which would be conducive to reducing the disruption of the esophageal mucosa and blood supply, and improve the match between anastomotic tissues, thereby lessening the chance of anastomosis-related complications caused by excessive tension. Third, most early anastomotic bleeding could be observed and sutured through the entry hole when using an LS for digestive tract reconstruction. Thus, an LS could be 
helpful for reducing the incidence of early anastomotic bleeding after TLTG. In brief, there are several promising inherent advantages to using IEJ with an LS instead of a CS.

There are several limitations in this study. First, this was a single-center retrospective study, and large-scale randomized controlled clinical trials are still lacking. Second, since the exact time of anastomosis was not recorded, its efficiency was indirectly estimated with the mean total operative time. Third, to achieve a better evaluation, patients with prior procedures, including abdominal surgery, preoperative chemoradiotherapy and combined surgery, were excluded from this study. Last, the short follow-up duration was insufficient to adequately assess the prognosis of gastric cancer.

\section{Conclusions}

TLTG is a safe and feasible procedure for treating UGC, with several advantages such as reducing the intraoperative time, intraoperative blood loss, and hospital stay compared with LATG. Moreover, the enjoyable space approach plus SPLT reconstruction is an appropriate comprehensive technique for completing TLTG. However, well-designed, large-scale randomized controlled clinical trials are needed to further demonstrate the real benefits of TLTG using the enjoyable space approach in conjunction with SPLT reconstruction.

\section{Acknowledgments}

The authors are thankful to Li-fang Qiu for collecting patients' follow-up data.

This study was sponsored by the Young and Middle-aged Teachers Education Scientific Research Project of Fujian Province (Grant number: JAT190556).

Xian-tu Qiu and Chang-yue Zheng contributed equally to this work.

\section{Conflict of interest}

The authors declare no conflict of interest.

\section{References}

1. Lee MS, Lee JH, Park DJ, et al. Comparison of short- and longterm outcomes of laparoscopic-assisted total gastrectomy and open total gastrectomy in gastric cancer patients. Surg Endosc 2013; 27: 2598-605.

2. Lin JX, Huang CM, Zheng CH, et al. Surgical outcomes of 2041 consecutive laparoscopic gastrectomy procedures for gastric cancer: a large-scale case control study. PLoS One 2015; 10 : e0114948.

3. Hao Y, Yu P, Qian F, et al. Comparison of laparoscopy-assisted and open radical gastrectomy for advanced gastric cancer: a retrospective study in a single minimally invasive surgery center. Medicine 2016; 95: e3936.

4. Komatsu S, Kosuga T, Kubota T, et al. Comparison of short- and long-term outcomes following laparoscopy and open total gastrectomy for gastric cancer: a propensity score-matched analysis. Am J Transl Res 2020; 12: 2225-33.

5. Kang SH, Cho YS, Min SH, et al. Intracorporeal esophagojejunostomy using a circular or a LS in totally laparoscopic total gastrectomy: a propensity-matched analysis. I Gastric Cancer 2019; 19: 193-201.

6. Shim JH, Yoo HM, Oh SI, et al. Various types of intracorporeal esophagojejunostomy after laparoscopic total gastrectomy for gastric cancer. Gastric Cancer 2013; 16: 420-7.

7. Inaba K, Satoh S, Ishida Y, et al. Overlap method: novel intracorporeal esophagojejunostomy after laparoscopic total gastrectomy. J Am Coll Surg 2010; 211: e25-9.

8. Lee IS, Kim TH, Kim KC, et al. Modified techniques and early outcomes of totally laparoscopic total gastrectomy with sideto-side esophagojejunostomy. J Laparoendosc Adv Surg Tech A 2012; 22: 876-80.

9. Ebihara Y, Okushiba S, Kawarada Y, et al. Outcome of functional end-to-end esophagojejunostomy in totally laparoscopic total gastrectomy. Langenbecks Arch Surg 2013; 398: 475-9.

10. Hong J, Wang YP, Wang J, et al. A novel method of self-pulling and latter transected reconstruction in totally laparoscopic total gastrectomy: feasibility and short-term safety. Surg Endosc 2017; 31: 2968-76.

11. Japanese Gastric Cancer Association. Japanese gastric cancer treatment guidelines 2014 (ver. 4). Gastric Cancer 2017; 20: 1-19.

12. Lam S, Tan E, Menezes A, et al. A comparison of the operative outcomes of D1 and D2 gastrectomy performed at a single Western center with multiple surgeons: a retrospective analysis with propensity score matching. World I Surg Oncol 2018; 16: 136.

13. Hagiwara A, Takahashi T, Sawai K, et al. Milky spots as the implantation site for malignant cells in peritoneal dissemination in mice. Cancer Res 1993; 53: 687-92.

14. Xie D, Liu L, Osaiweran H, et al. Detection and characterization of metastatic cancer cells in the mesogastrium of gastric cancer patients. PLoS One 2015; 10: e0142970.

15. Peeters KC, Marijnen CA, Nagtegaal ID, et al. The TME trial after a median follow-up of 6 years: increased local control but no survival benefit in irradiated patients with resectable rectal carcinoma. Ann Surg 2007; 246: 693-701.

16. van der Pas MH, Haglind E, Cuesta MA, et al. Laparoscopic versus open surgery for rectal cancer (COLOR II): short-term outcomes of a randomised, phase 3 trial. Lancet Oncol 2013; 14: 210-8.

17. Zheng MH, Zhang S, Feng B. Complete mesocolic excision: lessons from anatomy translating to better oncologic outcome. World J Gastrointest Oncol 2016; 8: 235-9.

18. Ouyang M, Luo Z, Wu J, et al. Comparison of outcomes of complete mesocolic excision with conventional radical resection 
performed by laparoscopic approach for right colon cancer. Cancer Manag Res 2019; 11: 8647-56.

19. Zheng CY, Dong ZY, Zheng LZ, et al. Laparoscopic D2 plus complete mesogastrium excision using the "enjoyable space" approach versus conventional D2 total gastrectomy for local advanced gastric cancer: short-term outcomes. Videosurgery Miniinv 2020; 15: 58-69.

20. Amin MB, Greene FL, Edge SB, et al. The eighth edition AJCC cancer staging manual: continuing to build a bridge from a population-based to a more "personalized" approach to cancer staging. CA Cancer J Clin 2017; 67: 93-9.

21. Chen K, Pan Y, Zhai ST, et al. Laparoscopic gastrectomy in obese gastric cancer patients: a comparative study with non-obese patients and evaluation of difference in laparoscopic methods. BMC Gastroenterol 2017; 17: 78.

22. Shen J, Ma X, Yang J, et al. Digestive tract reconstruction options after laparoscopic gastrectomy for gastric cancer. World J Gastrointest Oncol 2020; 12: 21-36.

23. Huang ZN, Huang CM, Zheng $\mathrm{CH}$, et al. Digestive tract reconstruction using isoperistaltic jejunum-later-cut overlap method after totally laparoscopic total gastrectomy for gastric cancer: short-term outcomes and impact on quality of life. World J Gastroenterol 2017; 23: 7129-38.

24. Xie D, Yu C, Liu L, et al. Short-term outcomes of laparoscopic D2 lymphadenectomy with complete mesogastrium excision for advanced gastric cancer. Surg Endosc 2016; 30: 5138-9.

25. Shen J, Cao B, Wang Y, et al. Prospective randomized controlled trial to compare laparoscopic distal gastrectomy (D2 lymphadenectomy plus complete mesogastrium excision, D2+CME) with conventional D2 lymphadenectomy for locally advanced gastric adenocarcinoma: study protocol for a randomized controlled trial. Trials 2018; 19: 432.

26. Wang FH, Shen L, Li J, et al. The Chinese Society of Clinical Oncology (CSCO): clinical guidelines for the diagnosis and treatment of gastric cancer. Cancer Commun 2019; 39: 10

27. Ko CS, Gong CS, Kim BS, et al. Overlap method versus functional method for esophagojejunal reconstruction using totally laparoscopic total gastrectomy. Surg Endosc 2021; 35: 130-8.

28. Murakami K, Obama K, Tsunoda S, et al. Linear or circular stapler? A propensity score-matched, multicenter analysis of intracorporeal esophagojejunostomy following totally laparoscopic total gastrectomy. Surg Endosc 2020; 34: 5265-73.

29. Park SH, Suh YS, Kim TH, et al. Postoperative morbidity and quality of life between totally laparoscopic total gastrectomy and laparoscopy-assisted total gastrectomy: a propensity-score matched analysis. BMC Cancer 2021; 21: 1016.

30. Liao T, Deng L, Yao X, et al. Comparison of the safety and efficacy between linear stapler and circular stapler in totally laparoscopic total gastrectomy: protocol for a systematic review and meta-analysis. BMJ Open 2019; 9: e028216.

31. Chen K, He Y, Cai JQ, et al. Comparing the short-term outcomes of intracorporeal esophagojejunostomy with extracorporeal esophagojejunostomy after laparoscopic total gastrectomy for gastric cancer. BMC Surg 2016; 16: 13.

32. Wei M, Wang N, Yin Z, et al. Short-term and quality of life outcomes of patients using linear or circular stapling in esophago- jejunostomy after laparoscopic total gastrectomy. J Gastrointest Surg 2021; 25: 1667-76.

Received: 14.12.2021, accepted: 14.01.2022. 